\title{
Self-regulatory strategy and executive control: implementation intentions modulate task switching and Simon task performance
}

\author{
Anna-Lisa Cohen • Ute C. Bayer • Alexander Jaudas • \\ Peter M. Gollwitzer
}

\begin{abstract}
Two tasks where failures of cognitive control are especially prevalent are task-switching and spatial Simon task paradigms. Both tasks require considerable strategic control for the participant to avoid the costs associated with switching tasks (task-switching paradigm) and to minimize the influence of spatial location (Simon task). In the current study, we assessed whether the use of a self-regulatory strategy known as "implementation intentions" would have any beneficial effects on performance in each of these task domains. Forming an implementation intention (i.e., an if-then plan) is a self-regulatory strategy in which a mental link is created between a pre-specified future cue and a desired goal-directed response, resulting in facilitated goal attainment (Gollwitzer in European Review of Social Psychology, 4, 141-185, 1993, American Psychologist, 54, 493-503, 1999). In Experiment 1, forming implementation intentions in the context of a taskswitching paradigm led to a reduction in switch costs. In Experiment 2, forming implementation intentions reduced the effects of spatial location in a Simon task for the stimulus specified in the implementation intention. Results supported the prediction that the need for high levels of cognitive control can be alleviated to
\end{abstract}

A.-L. Cohen $(\varangle) \cdot$ P. M. Gollwitzer $(\square)$

Department of Psychology, New York University,

6 Washington Place, 7th Floor, New York, NY 10003, USA

e-mail: alc312@nyu.edu

P. M. Gollwitzer

e-mail: peter.gollwitzer@nyu.edu

U. C. Bayer · A. Jaudas · P. M. Gollwitzer

University of Konstanz, Konstanz, Germany some degree by making if-then plans that specify how one responds to that critical stimuli.

\section{Introduction}

Human cognition and action is often triggered by the pursuit of a goal and the adoption of a plan in the hopes of achieving that goal. The picture increases in complexity when we consider that the cognitive system has processing restrictions such that attentional capacity is limited (Broadbent 1958). Executive control is the process by which the system or mind chooses a course of action that best satisfies the goal and it is synonymous with the construct executive function. This rather broad term is used to encompass a wide variety of cognitive processes such as dealing with novelty, planning, using strategies, monitoring performance, using feedback to modify performance, vigilance, and inhibiting irrelevant information (see Shallice 2005 for a review). Two domains in which executive control, and particularly failures of control are studied, are task switching and the Simon task. In this article, we examine a self-regulatory strategy known to enhance controlled processing in cognitive tasks, and we investigate whether this strategy will benefit performance in the context of task-switching and Simon task paradigms. Forming an implementation intention (i.e., an if-then plan) is a self-regulatory strategy in which a mental link is created between a pre-specified future situation and a desired goal-directed response, resulting in facilitated goal attainment (Gollwitzer 1993, 1999). We predict that the use of such a self-regulatory strategy can enhance performance in two task domains known for their high executive control requirements. 
Implementation intentions and control

Previous theories of goal pursuit emphasized conscious choice and guidance of behavior on a moment-tomoment basis (e.g., Bandura 1986). More recently, research has shown that mental representations of goals can become activated without an act of conscious will, such that subsequent behavior is then guided by these goals within the situational context faced by the individual (Bargh 1994). Automatic action initiation is the notion that established routines linked to a relevant context are released when the necessary conditions exist without the need for controlled or conscious intent (Bargh 1989). Bargh et al. (2001) showed that representations of goal-directed activity do not need to be put into motion by an act of conscious choice. In their study, Bargh and colleagues demonstrated that nonconsciously activated goals effectively guided action, enabling subjects to adapt to ongoing situational demands.

Furthermore, Gollwitzer $(1993,1999)$ suggested that forming a certain type of intention called an implementation intention is a self-regulatory strategy that alleviates the need for conscious control by delegating control to pre-specified environmental cues. In other words, implementation intentions link anticipated opportunities with goal-directed responses and thus commit a person to respond to a certain critical situation in a stipulated manner. Implementation intentions take the format of "If Situation X is encountered, then I will perform Behaviour Y!" They are to be distinguished from the more simple structure of a goal intention which has the format of "I intend to reach Z!", whereby $\mathrm{Z}$ may relate to a certain outcome or behavior to which the individual feels committed.

Implementation intentions are formed in the service of goal intentions and specify the when, where, and how a goal-directed response will be executed. Forming implementation intentions involves the selection of a critical future situation and it is assumed that implementation intentions lead to a heightened accessibility of the situational cue $(\mathrm{X})$, which in turn facilitates the detection of the situational cue in the environment. There is strong evidence for this perceptual readiness effect (Aarts, Dijksterhuis, \& Midden, 1999; Gollwitzer \& Schaal, 1998; Webb \& Sheeran, 2003). For example, in an experiment of Aarts et al. (1999) participants had the goal to pick up payment for their participation in an experiment in a location near a fire hose in the hallway. Participants who formed an implementation intention showed higher accessibility of the word "fire hose" in a lexical decision task (compared to participants holding the same goal but no implementation intention). Much like priming, this heightened accessibility makes it easier to detect the critical situation in the surrounding environment and readily attend to it even when one is in the midst of other ongoing activity. Furthermore, this heightened accessibility should facilitate the recall of the critical situation because a strong link has been formed between the two components (situation cue + response). Chasteen, Park, and Schwarz (2001) provided evidence that implementation intentions can significantly enhance older adults' prospective memory (i.e., ability to remember to execute an intention). They posited that creating an implementation intention caused behaviour to become reflexive, thus eliminating the need for conscious control once the prospective memory cue target was encountered. Therefore, encoding an implementation intention sets stored action schemas into a state of readiness such that, when the appropriate trigger conditions are satisfied, the intention can be executed without mediation of a conscious recollection of the intention. This research demonstrated that implementation intentions facilitated the attainment of goal intentions in a situation where it was easy to forget to act on them.

Our objective in the current paper is to examine the challenges of certain executive function tasks (i.e., switch task and Simon task) in that they create a conflict between bottom-up, stimulus-based control and top-down, intention-based processing. Both of these tasks require the participant to override some stimulusbased responding aspect of the task. For example, in Experiment 1 participants must override interference associated with having to switch to a new task rule and in Experiment 2 participants must ignore location of a stimulus in order to respond to its task-relevant features. In our research, we explore whether a self-regulation strategy which emphasizes top-down control (i.e., goal-based or plan-based control) can overcome stimulus-driven control so that better task performance emerges.

\section{Task switching}

A common paradigm that is used to measure cognitive control is task switching. In a typical experiment, the experimenter administers instructions that require the participant to internalize these instructions and adopt a task set in which several processes (e.g., sensory analysis, categorization, decisions, motor output) must be configured so that the task is successfully executed. Initial studies of task switching tried to explain switch costs in terms of solely one mechanism (e.g. Rogers \& Monsell, 1995). Currently, most authors now acknowledge that there may be more than one cause of switch 
costs and argument tends to centre on the exact nature or combination of those causes (see Monsell, 2003, for a review).

In Experiment 5 of Allport, Styles, and Hsieh (1994), the authors varied the duration between the response to the first stimulus and the presentation of the second stimulus. They discovered the surprising result in which residual switch costs remained even when the duration was extended to more than a second. That is, participants had more than enough time to prepare for the next response; however, the switch costs still remained. The authors interpreted this result as a reflection of a form of interference or persisting activation from the recently executed competing task. They referred to this phenomenon as task-set inertia in which task switching is more difficult when another task using the same stimuli had been executed in the last few minutes.

In contrast, Rogers and Monsell (1995) determined that this residual switch cost was due to the process of task-set reconfiguration which is driven by both endogenous (internally driven) and exogenous (externally driven) control. Endogenous control involves adopting a task set at will (a type of executive mechanism), whereas exogenous control is the case in which a stimulus evokes or activates a response. Rogers and Monsell (1995) examined the difficulty that endogenous control mechanisms have in overcoming exogenous activation of a task set. They concluded that there is a component of performance in task switching which cannot be overcome while anticipating the next appearance of the stimulus. That is, it is only when the next stimulus appears that the participant is exogenously triggered to perform the requirements of the task that is associated with that stimulus.

Hahn, Andersen, and Kramer (2003) examined the organization of preparatory processes and how they affect switch costs. Based on their results, they concluded that subjects' strategies may play an important role in the organization of preparatory processing in task switching.

\section{Simon task}

Another well-studied task that provides a measure of executive control is the Simon task. In this paradigm, the relevant stimulus dimension is a non-spatial dimension such as shape (e.g., square or circle) and the participant is asked to make a left key press if the stimulus is a square and a right key press if it is a circle (Simon, 1990). The location of the stimulus (left or right side of the computer screen) is the irrelevant stimulus dimension. The Simon effect refers to the phenomenon in which responses are faster when a stimulus' location (e.g., left side of computer screen) corresponds to the location of an assigned response (e.g., left computer key press). Performance is slower and more error prone when this stimulus-response correspondence is incompatible (e.g., stimulus is on left side of computer screen but requires a right computer key press). Subjects are instructed to respond on the basis of the relevant stimulus dimension and ignore the irrelevant dimension. However, numerous studies have shown that participants find it difficult to suppress or completely ignore the irrelevant dimension (summary by Lu \& Proctor, 1995). Even though stimulus position is irrelevant for the participant's task of identifying shape, it nevertheless interferes with performance.

The inability of individuals to overcome the influence of spatial location is seen as evidence of limitations on executive control over response activation. One widely accepted view of the Simon effect posits that there are two routes along which the stimuli are processed. One route processes the instructions to attend to the relevant dimension (e.g., shape) and this processing route is under intentional control. Another route is assumed to be automatic and it responds to the irrelevant dimension (e.g., spatial location). The automaticity of this response activation is due to long-term associations that are innate or have been over-learned during the lifetime. Therefore, when the relevant response to a stimulus (e.g., left key press) is in conflict with the spatial location of that stimulus (e.g., right side of computer screen) it leads to a response conflict which delays response execution (De Jong, Liang, \& Lauber, 1994; Hommel, 1993; Hommel, Proctor, \& Vu, 2004; Kornblum, Hasbroucq, \& Osman, 1990).

There are alternative accounts to this dual-route explanation based on evidence suggesting that automatic responses to spatial location of the stimulus may be under some control by the individual. For example, Mordkoff (1998) proposed an "information-gating" function, and Stürmer, Leuthold, Soetens, Schröter, and Sommer (2002) proposed a type of suppression mechanism based on findings that showed participants had some control over the influence of response activation. That is, performance in the Simon task was analyzed as a function of the preceding trial and they found that the Simon effect is robust after corresponding trials but not after noncorresponding trials. Therefore, it seems that the Simon effect is under some control of the individual such that they may suppress influence of the automatic effects of the spatial location information after noncorresponding trials. These findings provide some challenge to the dual route model explanation of the Simon effect. If participants exert 
some control over the influence of spatial location, it may be that the automatic component of the dualroute model is not purely automatic.

\section{The current research}

In Experiment 1, we examine the effect of implementation intentions within a task-switching paradigm. We predict that forming an implementation intention may function to alleviate the need to engage in effortful task-set reconfiguration processes thereby reducing potential switch costs. If automatic action initiation sets the associated response into motion, while circumventing effortful switching of task set, then performance should be less affected by the task that had to be performed in the preceding trial. Furthermore, we expected that there will not be increased distractor effects of the letter "E" within the digit-task because the implementation intention specifically pertains to the letter task. Previous research has shown that the benefit of implementation intentions tends to be very specific influencing only the situational cue + response that is stipulated in the implementation intention (Gollwitzer, 1999). In Experiment 2, we investigated the effect of implementation intentions within a Simon task paradigm. In Hommel et al. (2004), the authors state that the Simon task involves implementation of stimulus-response (S-R) translation rules that act in a reflexive way when the stimulus appears. For example, when a stimulus is detected, the system searches for some type of $\mathrm{S}-\mathrm{R}$ translation rule and then the correct response is activated. If we can provide a boost or advantage to this $\mathrm{S}-\mathrm{R}$ mapping through the adoption of an implementation intention, we may be able to reduce the influence of spatial location on incompatible trials.

\section{Experiment 1: implementation intentions enhance task switch performance}

Sohn and Anderson (2001) propose an ACT-R (adaptive control of thought-rational) model to explain task-switching costs. Their model assumes that information processing involves a sequence of production rule firings and each of these production rules involves "retrieving some declarative information, called chunks, to transform the current goal state" (p. 764, Sohn \& Anderson, 2001). They also suggest that the speed of retrieval of information depends on the level of activation of these rules. In a similar vein, implementation intentions are thought to lead to successful goal attainment based on heightened activation level of a situational cue which in turn eases retrieval of the associated response. Therefore, it may be that implementation intentions will facilitate task-switching performance because the necessary "chunk" of declarative information for performing the task will be highly activated through the formation of an implementation intention. Furthermore, Mayr and Kliegl (2003) stated that the stronger the association between the cue and task set, the faster the process of loading the necessary task set rules into working memory. These authors predict that stronger associations between cues and associated task sets should reduce switch costs. In the following experiment, we examine whether forming implementation intentions facilitates performance such that task-switching costs will be reduced.

We used the "alternating runs paradigm" (Rogers \& Monsell, 1995). Participants had to switch between two simple cognitive tasks in a predictable sequence (AABBAABB...). Participants in the implementation intention condition formed an implementation intention in which the stimulus "E" was specified as the critical stimulus. The following hypotheses were tested: (1) Forming implementation intentions will reduce switch costs but only when the critical stimulus is presented in the specific task (i.e., the letter "E" in the letter-task) and (2) There will be no increased distractor effect of the critical stimulus " $E$ " in the digit-task compared to the other letters $(\mathrm{U}, \mathrm{K}, \mathrm{M})$.

Method

\section{Participants}

Forty participants took part in this experiment $(20$ males, 20 females). All participants were recruited at the University of Konstanz. Age ranged from 20 to 34 years $(M=24.1$ years, $\mathrm{SD}=3.15)$. Participants were paid 3 Euro (approximately 3.6 USD). Thirty-two participants were right-handed, five were left-handed and one was ambidextrous (by self-report). Two participants refused to give information about their handedness. All participants had normal or corrected-tonormal eyesight.

\section{Design}

The design was a $2 \times 2 \times 2 \times 2$ factorial design with "intention" (goal intention vs. implementation intention) as the only between-subjects factor and "type of task" (letter-task vs. digit-task), "task switch" (yes vs. no), and "type of target" (neutral vs. critical) were all within-subject factors. 


\section{Material and procedure}

Participants were tested on an IBM-compatible Pentium computer with VGA graphics card using the Micro-Experimental Laboratory Professional software package (Schneider, 1988). A head rest was used to keep the viewing distance of $43 \mathrm{~cm}$ constant during the experiment. An outline of a square was presented on the computer screen which was separated into 4 smaller squares or quadrants with each subtending a visual angle of $6.7^{\circ}$ (see Fig. 1).

On each trial, a letter-number pair was presented in the center of one of these quadrants. Letters were sampled from the set $\mathrm{U}, \mathrm{E}, \mathrm{M}$ and $\mathrm{K}$, and numbers from the set of 5, 6, 7 and 8 . The stimuli characters extended a visual angle of $1.3^{\circ}$ vertically. The position of letter and number within each pair was randomized. The letter-number pairs remained on the screen until the participant pressed a key or until 5,000 ms had elapsed. The next character-pair was presented after a response stimulus interval (RSI) of $150 \mathrm{~ms}$. A relatively short RSI was chosen to make the task especially difficult based on the fact that $150 \mathrm{~ms}$ was the shortest RSI that Rogers and Monsell used in Experiment 3 in which a saving of the switch cost was observed as the R-S interval was increased from $150 \mathrm{~ms}$ to $600 \mathrm{~ms}$ (Rogers \& Monsell, 1995). In the current experiment, a $1,300 \mathrm{~Hz}$ signal tone was presented for a duration of $100 \mathrm{~ms}$ if the participant made a mistake and the subsequent ITI was extended to $1,400 \mathrm{~ms}$. If no response was made within $5,000 \mathrm{~ms}$, the trial was counted as an error.

When stimuli were presented in the upper two positions, a "letter task" had to be performed. In the letter task, participants had to decide as quickly and accurately as possible whether the letter of the letter-digit pair was a consonant or a vowel by pressing a corresponding key (the digit in was to be ignored). When the letter-digit pair was presented in the lower two positions, a "digit task" had to be performed in which participants had to decide as quickly and accurately as possible whether the digit was odd or even (the letter had to be ignored). In each trial, the letter-digit pair was presented in the next quadrant in a predictably clockwise pattern. Thus, if the letter-digit pair appeared in the upper right quadrant, it would appear

Fig. 1 Schematic of the display in Experiment 1 in the alternating runs task (As the stimulus is presented in the upper row, the letter task had to be performed which means the letter "E" had to be categorized as a vowel) in the lower right quadrant in the subsequent trial. So the sequence of the tasks was AABBAABB... (Aswitch, A-repetition, B-switch, B-repetition). This presentation sequence ensured that working memory demands as well as arousal level would be similar in switch and non-switch trials (Rogers \& Monsell, 1995). The stimulus position (upper or lower half) indicated which of the two tasks were to be performed. Therefore, the position of the targets served as a cue for the participant and made the task of switching attention between the two tasks fully predictable. The dependent variables were reaction time performance and error rate.

Participants received written instructions. They were instructed to decide as fast and as accurately as possible whether the relevant stimulus was a vowel or a consonant (letter-task) and whether a number was an odd or even digit (digit-task). Participants completed six practice blocks ( 48 trials per block) to familiarize themselves with the tasks. After completing the practice blocks, the factor intention was manipulated. In this task, half of the participants furnished one subgoal of the task with an implementation intention and the other half formed a goal intention. All instructions were given in written form. Subjects in the goal condition read the following sentence: "It's your task to react as fast as possible to the stimuli and at the same time make no errors." Subjects in the implementation intention condition additionally had to read the following sentence: "And if there appears an "E" in the upper row, then I'll press the right button especially fast." Furthermore, subjects were asked to internalize the implementation intention by saying it aloud three times.

Therefore, we predicted that forming an implementation for this one sub-goal should benefit performance compared to other sub-goals. The main portion of the experiment consisted of 8 blocks of 48 trials (384 trials in total). At the end of each block, participants were reminded of their goal and implementation intention and a feedback display indicated their mean reaction time. The experiment lasted approximately $40 \mathrm{~min}$.

\section{Results and discussion}

All incorrect responses were removed from reaction time analyses and latencies beyond three standard deviations (SDs) of the cell mean or less than $300 \mathrm{~ms}$ were also deleted. The result was the overall removal of $3.6 \%$ of the data. As the critical stimulus "E" plays a different role within the two tasks (critical stimulus vs. distractor), the data were analyzed separately for the letter and digit task. 


\section{Letter-task}

Reaction time performance was evaluated using a 2 (type of intention: goal intention, implementation intention) $\times 2$ (task switch: yes, no) $\times 2$ (Type of stimulus: neutral, critical) mixed factorial analysis of variance (ANOVA) with repeated measures on the second and third factors. There was a significant main effect for the factor "Task Switch", $F(1,38)=185.4$, $P<0.001$, showing that RTs were slower after switch trials $(M=1,090 \mathrm{~ms}, \mathrm{SD}=240.7)$ than after nonswitch trials $(M=737 \mathrm{~ms}, \mathrm{SD}=164.1)$. This main effect was qualified by an interaction between this factor and "Type of Intention", $F(1,38)=8.70, P<0.01$, indicating that switch costs were substantially smaller in the implementation intention condition $(M=277 \mathrm{~ms}$, SD $=163.3)$ than in the goal intention condition $(M=$ $430 \mathrm{~ms}, \mathrm{SD}=165.2)$. The main effect for the factor "Type of Stimulus" was also significant, $F(1,38)=18.2$, $P<0.001$, showing that reactions to neutral items $(M=949 \mathrm{~ms}, \mathrm{SD}=191.1)$ were slower than critical items $(M=878 \mathrm{~ms}, \mathrm{SD}=202.4)$. This main effect was qualified by an interaction between this factor and "Type of Intention", $F(1,38)=25.2, P<0.001$. Planned comparisons in the goal intention condition revealed no significant difference $(P=0.60)$ between neutral and critical stimuli in response times (difference score: $M=12 \mathrm{~ms}, \mathrm{SD}=102.5$ ). However, in the implementation intention condition, this difference was significant, $t(19)=6.48, P<0.001$, showing that responses to the critical stimuli were comparatively faster $(M=153 \mathrm{~ms}$, $\mathrm{SD}=105.5)$. The interaction of all three factors was not significant, $F(1,38)=0.13, P=0.72$. In terms of error rates, there was only a significant main effect for "Task Switch", $F(1,38)=23.7, P<0.001$, indicating that the error rate was $2.7 \%$ in non-switch conditions compared to an error rate of $6.7 \%$ under switch conditions. See Fig. 2.

\section{Digit-task}

Analysis of reaction times revealed only a significant main effect for the factor "Task Switch", $F(1,38)=$ 207.9, $P<0.001$. Performance was faster in nonswitch trials $(M=733 \mathrm{~ms}, \mathrm{SD}=153.1)$ compared to switch trials $(M=1,227 \mathrm{~ms}, \mathrm{SD}=292.1)$. Analysis of error rates yielded a main effect for the factor "Task Switch", $F(1,38)=78.7, P<0.001$, indicating a larger proportion of errors in the non-switch trials $(4.2 \%)$ compared to switch trials $(8.7 \%)$. This main effect was qualified by a marginally significant interaction between this factor and "Type of Intention", $F(1,38)=$ 3.76, $P=0.06$. Under non-switch conditions, the error
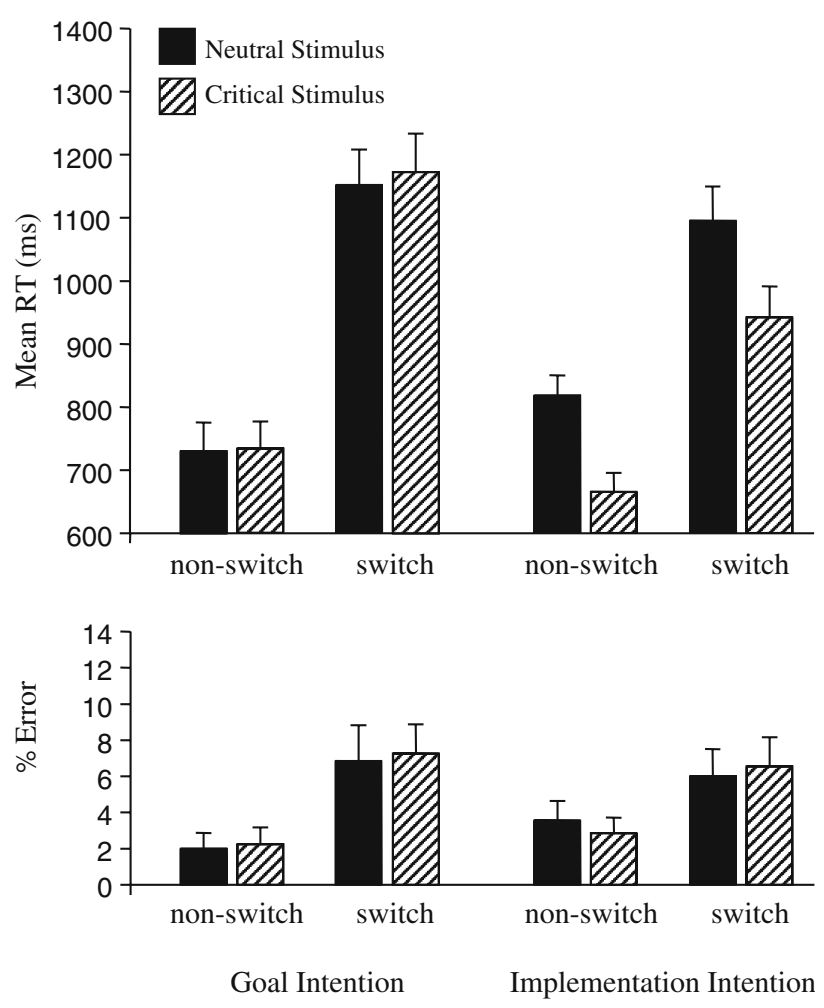

Fig. 2 Mean reaction time performance for correct trials and error rates in Experiment 1 in the letter-task as a function of task switch, type of stimulus, and type of intention. Bars represent standard error (SE)

rates in the goal intention condition were $2.0 \%$ higher compared to the implementation intention condition, whereas this difference reached $4.0 \%$ under switch conditions. See Fig. 3.

In the letter task, results were similar to those found by Rogers and Monsell (1995) in that reaction time switch costs $(353 \mathrm{~ms})$ and error rates $(4.0 \%)$ were quite substantial. However, reaction time costs in the current experiment were $116 \mathrm{~ms}$ higher than those found in Rogers and Monsell's Experiment 1 (i.e., $237 \mathrm{~ms}$ ) and error rates were lower in our study. These differences may be attributable to subjects adopting a different response criterion in our experiment due to the manipulations of goal and implementation intentions. The interaction of the factors "Task Switch" and "Type of Intention" was due to the fact that in the implementation intention condition switch costs were lower ( $277 \mathrm{~ms}$ ) compared to switch costs in the goal intention condition $(430 \mathrm{~ms})$. There was no interaction of all three factors because in the implementation intention condition reaction times on the critical stimulus were reduced not only in switch trials but also in non-switch trials (see Fig. 2). If reaction times in switch trials for the critical stimulus are compared to reaction times for 

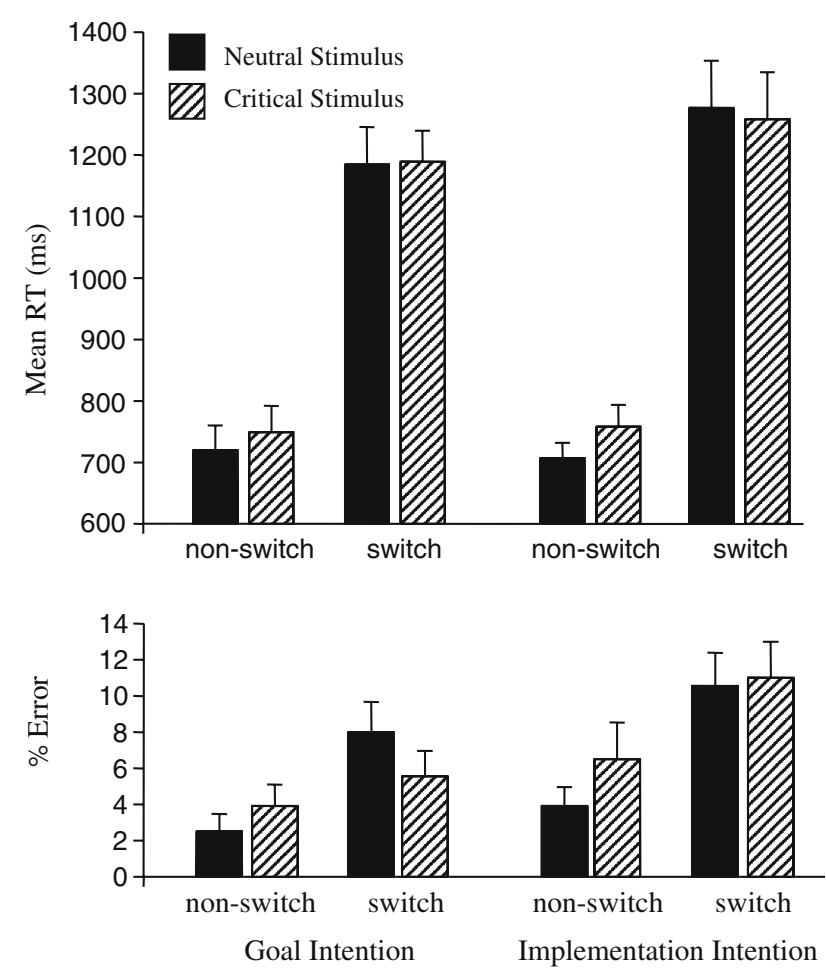

Fig. 3 Mean reaction time performance in Experiment 1 for correct trials and error rates in the digit-task as a function of task switch, type of stimulus, and type of intention. Bars represent SE

the other stimuli, a significant reduction of switch costs is observed, $t(19)=3.98, P<0.001$.

Concerning the non-switch trials it seems that there is a kind of tradeoff between the implementation intention condition and the goal condition. In the goal condition, planned comparisons revealed no significant difference between neutral and critical stimuli. In the implementation intention condition, there was a significant difference, $t(19)=7.24, P<0.001$. Therefore, it appears that the heightened activation of the critical stimulus (the letter "E") leads to facilitation in processing for this stimulus but at a cost of the remaining neutral stimuli. This result suggests that forming implementation intentions benefits only the critical cue that was specified in the formation of the implementation intention. It could be that participants may have interpreted the implementation intention manipulation as meaning that the neutral stimuli were somehow "less important" and thus devoted less effort to processing these stimuli. Another possible interpretation is that our manipulation of specifying the target "E" in an implementation intention led to a type of arousal every time this target appeared. For example, Meiran and Chorev (2005) showed that increased alertness leads to a reduction of residual switch costs. However, this explanation is likely not sufficient as an explanation as switch costs were relatively large in our experiment compared to those observed by Meiran and Chorev (2005).

For digit-task performance, similar to results found by Rogers and Monsell (1995), there were substantial switch costs in the digit-task in terms of reaction times $(M=494 \mathrm{~ms}, \mathrm{SD}=217.9)$ and error rates $(4.6 \%)$. Similar to findings observed in the letter task, switch costs were higher and error rates were lower compared to Rogers and Monsell's results (RT: $M=289 \mathrm{~ms}$; Error rate $=6.1 \%$ ). As the critical stimulus served as a distractor in the digit-task, we were most interested in the comparison between neutral and critical stimuli, however, there were no reliable differences. We interpret this result to mean that the effect of the implementation intention manipulation was limited to the lettertask. Although the interaction of "Task Switch" and "Type of Intention" was marginally significant, it suggests that there may be a type of preparation for the upcoming trial. That is, the trial that follows a nonswitch trial in the digit-task is a switch trial in the lettertask in which the critical stimulus is relevant again. In the implementation intention condition in non-switch trials, the error rates for the critical stimulus are higher compared to the neutral stimuli. It may be that subjects in the implementation intention condition know that the upcoming trial will be the switch trial in the letter task. Accordingly, subjects may begin preparing for the letter task too early, so that accuracy in the digit task is reduced especially when the critical stimulus is the distractor.

In summary, we obtained substantial switch costs in terms of response latencies and error rates and therefore replicated the basic findings of Rogers and Monsell (1995, Experiment 1). As mentioned previously, reaction time costs in the current experiment were higher than those observed by Rogers and Monsell (1995). One limitation of the current experiment is that there was no neutral group. Some might argue that instructions for both conditions increased working memory load leading to higher costs and that the implementation intention manipulation was simply less harmful than the goal intention condition. However, we think such an interpretation is implausible because previous research on implementation intentions has tested the possibility these manipulations increase cognitive load and found no evidence (e.g., Cohen, Jaudas, \& Gollwitzer, 2005). Forming implementation intentions led to a reduction of response times for the critical stimulus in the letter task. Furthermore, implementation intentions did not cause costs in the digittask except for a marginally significant increase of error rates under switch conditions which could be 
interpreted as a kind of preparation effect for the upcoming task. These results show that subjects' strategies are important in determining task switching performance. In the next experiment, we were interested in testing our manipulation of implementation intentions in a different paradigm that also measured executive controlled processing.

\section{Experiment 2: implementation intentions and reduction of the simon effect}

In Experiment 2, participants performed a Simon task (Simon, 1990) and the specific paradigm was based in part on an experimental design used by Hommel (1993, Experiment 1). Participants had to decide whether the pitch of a tone (that occurred either on the left or right side of the participant) was high or low by pressing a left or right key. Thus, participants had to ignore the spatial location (left or right) of the tone especially when the location of the tone (e.g., right) and key press (e.g., left) were incongruent. The difficulty of overcoming the influence of spatial location is seen as evidence of limitations on executive control over response activation. Therefore, we expected that subjects in the goal intention condition would experience a Simon effect. In contrast, participants in the implementation intention condition formed an implementation intention establishing a link between an incongruent stimulus and the respective correct response. As information processing and action initiation via implementation intentions is assumed to be automatic, we predicted that this should lead to a reduction of the Simon effect for subjects in the implementation intention condition.

\section{Method}

\section{Participants}

Thirty-six participants took part in this experiment (26 males, 10 females). Age ranged from 20 to 42 years $(M=23.8$ years, $\mathrm{SD}=4.37)$. Again, all participants were recruited at the University of Konstanz and participants were paid 5 Euro (approximately 6 USD). Thirty-three participants were right-handed, three were left-handed. All participants had normal or corrected-to-normal eyesight.

\section{Design}

The design was a $2 \times 2 \times 2$ within-subjects factorial design with "intention" (goal intention vs. implementation intention), "congruency" (congruent vs. incongruent) and "type of target" (neutral vs. critical) as within-subject factors.

\section{Material and procedure}

Participants were tested on an IBM-compatible Pentium computer with a VGA graphics card and a Soundblaster 16 (TM) audio card using the MicroExperimental Laboratory Professional software package (Schneider, 1988). Subjects sat directly in front of the computer monitor. A head rest was used to keep the viewing distance of $93 \mathrm{~cm}$ consistent throughout the experiment. On each side of the computer monitor a loudspeaker was positioned. The distance between the center of the screen and the loudspeakers was $42 \mathrm{~cm}$. A high and a low pitch tone were used as stimuli and the frequency of the tones was 200 and $500 \mathrm{~Hz}$, respectively. The volume for both tones was adjusted to $55 \mathrm{~dB}$. The tones were presented on either the left or right side of the subject through the loudspeakers. On each trial a fixation cross was presented for $150 \mathrm{~ms}$ followed by a blank screen for $850 \mathrm{~ms}$. Then the stimulus tone was presented for a maximum duration of $1,000 \mathrm{~ms}$.

During this time period, subjects had to make their response by pressing either the " $y$ " key or the "." key on the computer keyboard using the index finger of each hand. ${ }^{1}$ Key assignment was counterbalanced across participants. If subjects did not respond within $1,000 \mathrm{~ms}$, the feedback "too slowly" was presented on the screen for $500 \mathrm{~ms}$. If subjects pressed the wrong key, the feedback "wrong key" was presented on the screen for $500 \mathrm{~ms}$. The inter-trial interval was set to 1,500 ms. Each portion of the experiment began with 12 practice trials that were excluded from data analysis.

As opposed to Experiment 1, we used a within-subjects manipulation of intention. The experiment consisted of two blocks with each block having 120 trials. In the first part of the experiment, subjects received written instructions for the goal intention condition. They were told to decide as fast and as accurately as possible whether the pitch of the presented tone was high or low by pressing the respective key on the computer keyboard. In this way, the following goal was administered: "I'll respond to the tones by pressing the respective button as fast as possible." After the first part of the experiment subjects fostered this goal intention by forming the following implementation intention: "And if I hear the low tone on the left side, then I'll press the right button especially fast." For partici-

\footnotetext{
${ }^{1}$ On a German computer keyboard, these keys are located symmetrically on the left and right sides of the keyboard.
} 
pants in the implementation intention condition, assignment of pitch and location were counterbalanced. That is, the possible situational cue + response pairings (e.g., low tone left + press right; high tone left + press right; low tone right + press left; high tone right + press left) were counterbalanced across participants.

Instructions for the goal and implementation intention were not counterbalanced across blocks because it is unlikely that subjects would be able to adopt an implementation intention in the first block and then not use this strategy in the second block. Furthermore, we were most interested in comparing the critical situation specified in the implementation intention as one of two possible incongruent combinations of response and spatial location. The experiment lasted about $35 \mathrm{~min}$ and the dependent variables were reaction time and error rates.

\section{Results and discussion}

All incorrect responses were removed from reaction time analyses and latencies beyond 3 SDs of the cell mean or less than $300 \mathrm{~ms}$ were also deleted. The result was the overall removal of $5.1 \%$ of the data.

\section{Reaction times}

Reaction time performance was evaluated using a 2 (intention: goal intention, implementation intention) $\times 2$ (congruency: congruent, incongruent) $\times 2$ (type of target: neutral, critical) repeated measures analysis of variance (ANOVA). There was a significant threeway interaction, $F(1,35)=6.36, P<0.05$. Analyzing the goal intention trials only led to a highly significant main effect for the factor "congruency", $F(1,35)=99.7$, $P<0.001$. Responses were faster on congruent $(M=362 \mathrm{~ms}, \mathrm{SD}=52.6)$ than on incongruent trials $(M=406 \mathrm{~ms}, \mathrm{SD}=58.4)$. Therefore the Simon effect was about $44 \mathrm{~ms}$ (i.e., the difference between congruent and incongruent trials). In contrast, performance for implementation intention trials revealed a significant interaction between the factors "congruency" and "type of target", $F(1,35)=14.0, P<0.001$. Thus, only the neutral stimulus showed the Simon effect, $t(35)=$ 3.78, $P<0.001$, with congruent trials $(M=376 \mathrm{~ms}$, $\mathrm{SD}=65.6)$ faster compared to incongruent trials $(M=$ $398 \mathrm{~ms}, \mathrm{SD}=53.2)$. For the critical stimulus, planned comparisons revealed no significant difference in reaction times, $t(35)=0.68, n s$ (congruent trials $M=367 \mathrm{~ms}$, $\mathrm{SD}=68.0$, incongruent trials $M=361 \mathrm{~ms}, \mathrm{SD}=59.0)$.

\section{Error rates}

The interaction of the factors "intention" and "congruency" was significant, $F(1,35)=11.4, P<0.01$, indicating that subjects made more errors in the implementation intention trials than goal intention trials when responding to congruent stimuli, $t(35)=4.64, P<0.001$, but for the incongruent stimuli this comparison was not significant. Analyzing the goal intention trials separately led to a highly significant main effect for the factor "congruency", $F(1,35)=26.5, P<0.001$, demonstrating that performance was more error prone for incongruent trials $(M=8.31 \%, \mathrm{SD}=7.12)$ than for congruent trials $(M=2.21 \%, \mathrm{SD}=2.58)$. The actual Simon effect was $6.1 \%$. Similar to the reaction time analyses, there was no interaction between the factors "congruency" and "type of target." However, for the implementation intention trials there was a significant main effect for the factor "congruency", $F(1,35)=4.36, P<0.05$, and this main effect was qualified by a marginally significant interaction between this factor and "type of target", $F(1,35)=3.09, P=0.08$. For the neutral stimuli, planned comparisons revealed a significant Simon effect, $t(35)=2.95, \quad P<0.001 \quad$ (congruent trials $M=4.83 \%, \mathrm{SD}=3.99$, incongruent trials $M=7.61 \%$, $\mathrm{SD}=6.22$ ), but for the critical stimuli there was no significant difference in error rates, $t(35)=0.8, n s$. See Table 1.

The present results provide the first demonstration that it is possible to eliminate the Simon effect by means of forming implementation intentions. The

Table 1 Mean reaction times (RTs) and percentages of error (PEs) in Experiment 2 as a function of the factors intention, congruency and type of target

\begin{tabular}{|c|c|c|c|c|c|c|c|c|c|c|c|c|c|c|c|c|}
\hline & \multicolumn{8}{|c|}{ Goal intention } & \multicolumn{8}{|c|}{ Implementation intention } \\
\hline & \multicolumn{4}{|c|}{ Congruent } & \multicolumn{4}{|c|}{ Incongruent } & \multicolumn{4}{|c|}{ Congruent } & \multicolumn{4}{|c|}{ Incongruent } \\
\hline & \multicolumn{2}{|l|}{ RT } & \multicolumn{2}{|l|}{ PE } & \multicolumn{2}{|l|}{ RT } & \multicolumn{2}{|l|}{$\mathrm{PE}$} & \multicolumn{2}{|l|}{ RT } & \multicolumn{2}{|l|}{$\mathrm{PE}$} & \multicolumn{2}{|l|}{ RT } & \multicolumn{2}{|l|}{ PE } \\
\hline & $M$ & $\mathrm{SD}$ & $M$ & SD & $M$ & SD & $M$ & SD & $M$ & $\mathrm{SD}$ & $M$ & $\mathrm{SD}$ & $M$ & SD & $M$ & $\mathrm{SD}$ \\
\hline Neutral & 360 & 51.6 & 1.83 & 2.15 & 407 & 57.9 & 8.31 & 9.27 & 376 & 65.6 & 4.83 & 3.99 & 398 & 53.2 & 7.61 & 6.22 \\
\hline Critical & 364 & 57.1 & 2.58 & 3.62 & 405 & 62.2 & 8.31 & 6.64 & 367 & 68.0 & 5.22 & 4.76 & 361 & 59.0 & 6.11 & 5.16 \\
\hline
\end{tabular}


reaction times and error rates for the goal intention trials resemble the results from Hommel (1993, Experiment 1, Group 1). Under goal intention conditions, a highly significant Simon effect was found for reaction times (44 ms) and error rates $(6.1 \%)$. The respective values in Hommel's experiment were $73 \mathrm{~ms}$ and $7.0 \%$. In their review, Lu and Proctor (1995) reported Simon effects of a magnitude of $20 \mathrm{~ms}$ up to $70 \mathrm{~ms}$. As there were no significant differences in reaction times or error rates concerning the type of the stimulus (neutral vs. critical), the spatial Simon effect could be replicated in the first part of the experiment (goal intention trials). In the second part of the experiment (implementation intention trials), one of the incongruent stimuli was supported by forming an implementation intention (critical stimulus). For the neutral stimuli which were not specified in the implementation intention, a Simon effect for reaction times (22 ms) and error rates (3.6\%) was obtained. Compared to the first part of the experiment, the Simon effect was weaker probably due to accumulated practice-nevertheless it was still highly significant. Most importantly, for the performance on the critical stimulus, no Simon effect was observed on reaction times or error rates in the second part of the experiment (i.e., on the implementation intention trials). See Fig. 4.

Next, we examined reaction time distribution analyses using the Vincentization method of Ratcliff (1979). We were interested in analyzing whether the observed effects were bound to a certain part of the distribution only. For example, Hommel (1997) has shown that the Simon effect tends to be stronger in the faster reaction times compared to slower reaction times. We conducted an ANOVA in which we represented each condition within subject by three quartiles (e.g., 25, 50, and 75th percentiles) grouping RTs from fastest to slowest. Then we entered quartile as an additional independent variable in the ANOVA. We were interested whether the Simon effect would vary as a function of quartile. More importantly, we wanted to examine whether the benefits of implementation intentions would be bound to one of the quartiles. We conducted a 2 (goal: goal, implementation intention) $\times 2$ (congruency: congruent, incongruent $) \times 2$ (stimuli: critical vs. neutral) $\times 3$ (quartile: 1, 2, 3) repeated measures ANOVA. Results revealed that the factor quartile did not interact with any of the other factors (all $P_{\mathrm{s}}>0.05$ ). Inspection of Figs. 5 and 6 show that the Simon effect was observed in the goal condition for both critical and neutral stimuli and for neutral stimuli in the implementation intention consistently across the three quartiles. There was no Simon effect in the implementation intention condition for the critical stimulus (see lower panel of Fig. 6). To
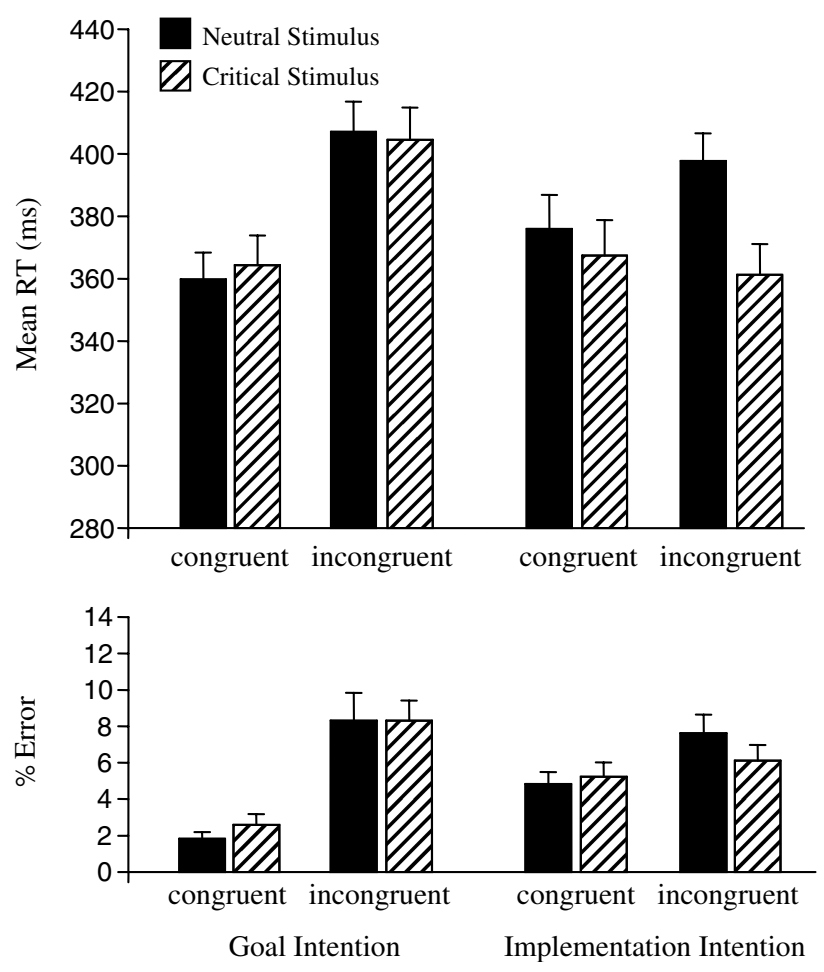

Fig. 4 Mean reaction time performance in Experiment 2 for correct trials and error rates as a function of intention, congruency, and type of target. Bars represent SE

summarize, in contrast to previous findings (e.g., Hommel, 1997), the Simon effect did not vary according to quartile and it was completely eliminated when participants furnished their performance with an implementation intention that specified a critical stimulus. Results showed that the Simon effect did not vary as a function of quartile nor was the benefit of implementation intentions bound to one of the quartiles.

The overall pattern of results supports our prediction but one further aspect of the results is noteworthy. Concerning the congruent stimuli, subjects made more errors in the implementation intention trials compared to the goal intention trials $(2.2$ vs. $5.0 \%)$. It is important to stress that this was only a main effect for "intention" that was not further qualified by "type of target." A possible explanation could be that specifying an incongruent stimulus in the implementation intention led subjects to focus on the incongruent stimuli and thereby led to some neglecting of the congruent stimuli. Thus, there may be some attention related costs caused by forming implementation intentions.

It is interesting to note that results from Experiment 1 reflected an overall switch-cost reduction in the implementation intention group. However, contrary to our predictions, this reduction was not specific of the critical 
Fig. 5 Means of individual reaction time quartiles for performance in the goal condition for the neutral stimulus (upper panel) and critical stimulus (lower panel) as a function of congruence (straight lines) or incongruence (dotted lines)

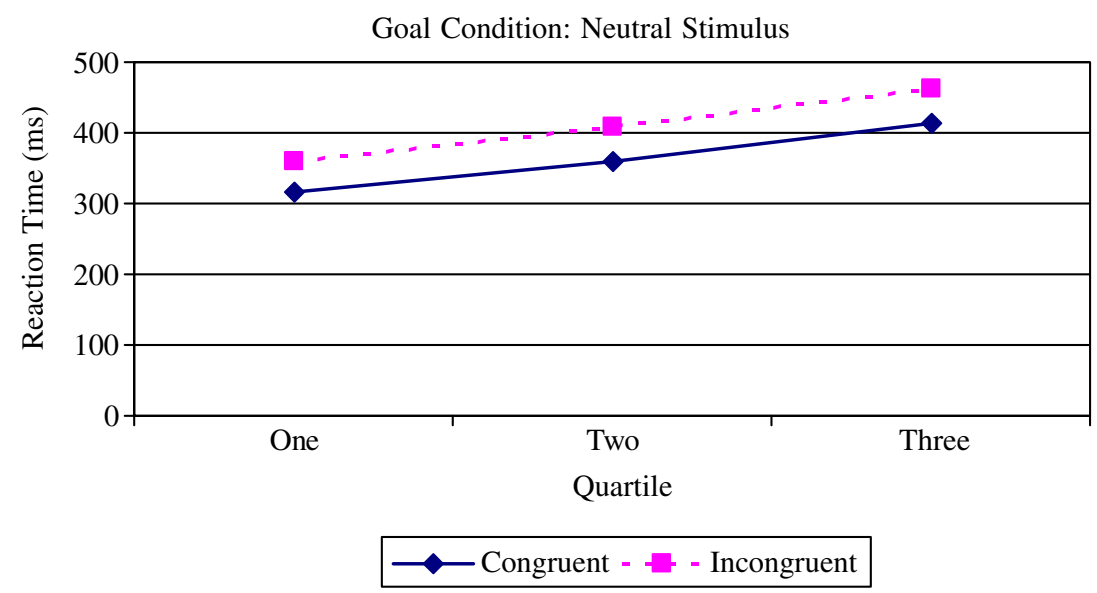

Goal Condition: Critical Stimulus

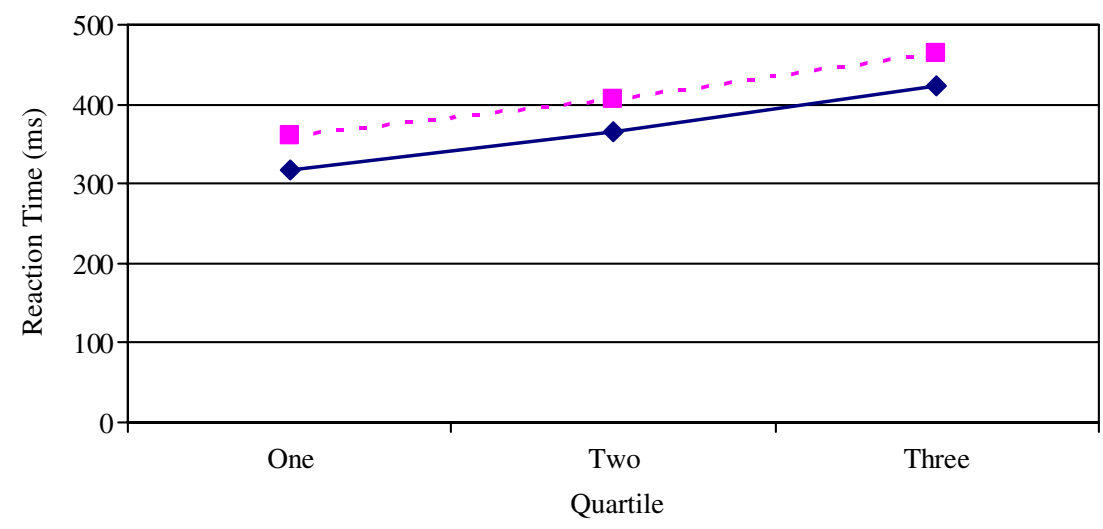

item specified in the implementation intention (i.e., "E"). However in Experiment 2, the effect of implementation intentions were specific in terms of influencing only the critical stimuli specified in the implementation intention. A possible explanation may lie in the fact that the implementation intention in Experiment 1 was slightly more complex than in Experiment 2. It required subjects to keep in mind the situational cue "E" and this critical stimulus had to be maintained in the context of three other possible distractors (e.g., U, M, K). In contrast, in Experiment 2 the subject had to distinguish the critical stimulus, if it was a low tone, from only one other possible distractor (a high tone). Thus, it may be that the increased complexity of the implementation intention in Experiment 1 led to a more generalized reduction of costs compared to the more specific effects obtained in Experiment 2.

\section{General discussion}

The aim of the present research was to examine the effect of implementation intentions in two different task domains that are known to require high levels of executive control. More precisely, we predicted that implementation intentions would lead to heightened activation of situational cues within the tasks thus facilitating the initiation of the intended responses. Overall, the results of the present studies confirmed these predictions. In Experiment 1, forming implementation intentions in the context of a task-switching paradigm led to an overall reduction in switch costs in the implementation intention compared to the goal condition. In Experiment 2, the automatic effects of spatial location in the Simon task were reduced when participants furnished one of the sub-goals with an implementation intention. It should be noted that results of the lettertask in Experiment 1 indicate an overall switch-cost reduction in the implementation intention group with respect to the goal intention group. In contrast to predictions, this reduction was not specific to the critical item (i.e., "E") specified in the implementation intention. However, implementation intentions did result in reduced absolute reaction time performance (i.e., faster RTs for critical items versus neutral items). Implementation intentions produced a general benefit 
Fig. 6 Means of individual reaction time quartiles for performance in the implementation intention condition for the neutral stimulus (upper panel) and critical stimulus (lower panel) as a function of congruence (straight lines) or incongruence (dotted lines)
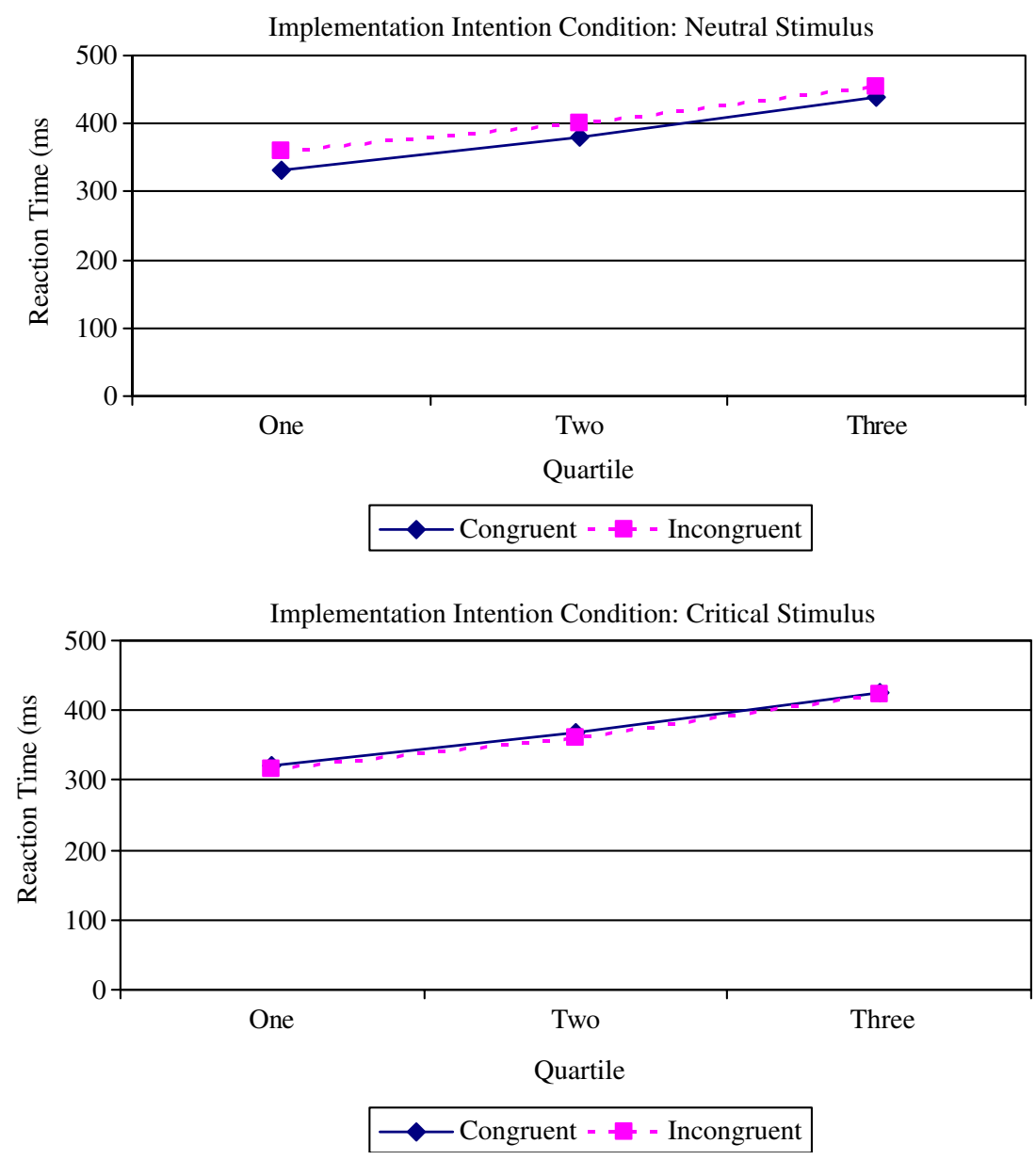

in Experiment 1 and a specific benefit to the critical stimulus in Experiment 2. These results indicate that implementation intentions may have general or specific effects depending on the task requirements. The link between the situational cue and response may have been more easily achieved in Experiment 2 leading to a benefit for the critical stimulus whereas this link may have been less well-established in Experiment 1 leading to a more generalized benefit to all stimuli within the implementation intention condition.

Mayr and Kliegl (2003) proposed a two-stage theory in which task-set selection was determined by: (a) cuedriven retrieval of task rules from long-term memory, and (b) the automatic application of rules to a particular stimulus situation. Therefore, the first stage is a retrieval stage that is concerned with cue-driven retrieval of rules from long-term memory into working memory. The second stage is an application stage in which task rules are applied in a fairly automatic fashion. Mayr and Kliegl demonstrated that most of the total switch costs were not due to task-switching processes per se but rather to a change in cue-associated processing. For example, the switch costs are not due to configuring the system for upcoming task demands but, upon seeing the cue, the cost is due to loading task rules from long-term memory into working memory. If we decompose the structure of implementation intentions into their component parts, we can see how encoding an implementation intention may strengthen each stage as described by Mayr and Kliegl (2003). The first portion of an implementation intention (i.e., "If situation $\mathrm{x}$ arises") is focused on specifying a situational cue. It focuses on the "I must do something when I encounter x." Therefore, this first half of the implementation intention may serve to establish the retrieval stage of the two process model. The second portion of the implementation intention (i.e., "Then I will perform response $y$ !") focuses on application of the associated response, and therefore it may serve to strengthen the application stage of the two-process model. As Mayr and Kliegl (2003) state "During a switch, participants use the cue to retrieve the task set. Optimally, the task set specifies the necessary S-R associations. The stronger the association between the cue and the task set and between the stimuli and responses, the faster this process of loading the adequate information 
into working memory should be executed" (p. 364). Numerous studies have shown that the formation of an implementation intention links the situational cue to the associated behavior (see Gollwitzer \& Sheeran, 2006, for review). This delegates the initiation of the intended behavior or response to the situational cue (Gollwitzer \& Schaal, 1998).

Although forming implementation intentions reduced switch costs considerably, it did not completely eliminate switch costs. It may be that an implementation intention facilitates the process of loading the adequate information into working memory once the cue is encountered. However, as Rogers and Monsell (1995) conclude, there is a limit to the preparation or reconfiguration that can be initiated endogenously. Thus, it may be that the exogenous trigger of a stimulus must be encountered for the participant to complete the process of reconfiguration (Rogers \& Monsell, 1995). There is increasing focus within the field of task switching regarding the way in which stimuli can trigger task sets, potentially in conflict with the task set that participants intend to adopt (e.g., Wylie \& Allport, 2000, Experiment 5; Waszak et al., 2003). It may be that this phenomena is fundamentally similar to implementation intentions in the sense that subjects delegate control of their behavior to the environment so that when a stimulus appears that was previously associated with task A, task A becomes activated, even if the subject was actually intending to perform task B. However, our data suggest that environmentally cued triggering of task sets may be independent of the effect of implementation intentions, as in our experiment forming implementation intentions seemed to affect switch and non-switch trials equally, and the effect of the target versus nontarget letter was confined to the letter task. This finding contrasts with the stimuluscued effects that have been reported in the literature before, which are typically larger on switch than nonswitch trials, and where primed stimuli affect performance in both tasks (i.e., facilitate performance in the task with which they have been associated, and impair performance in the other task). Nevertheless, we demonstrated that the intentional use of a self-regulatory strategy such as implementation intention achieved a reduction in switch costs which is comparable to reductions observed in other studies that used manipulation of probabilities (Dreisbach, Haider, \& Kluwe, 2002) or increased practice (Mayr \& Kliegl, 2003).

As discussed earlier, the Simon task is thought to involve two response selection processes: automatic activation and intentional translation ( Vu, Proctor, \& Urcuioli, 2003). This dual route explanation has been called into question by those who showed that the automatic response selection may be under some intentional control (e.g., Stürmer, Leuthold, \& Sommer, 1998). Therefore, some evidence suggests that the Simon effect may be under some control of the individual such that research participants may suppress the influence of the automatic effects of the spatial location information. However, Hommel et al. (2004) argue that even if gating or suppression of automatic influences is a viable explanation, this process may not be under individual control. Therefore, it is not necessary to dismiss a dual process model of the Simon effect. Hommel et al. (2004) propose an explanation that describes Simon task performance in terms of feature integration or event files. Their idea is that stimuli and responses that occur in time have features that are spontaneously integrated into a common transient representational structure or "event file" (Hommel et al., 2004). In a standard Simon task, there are $2 \times 2$ or four possible combinations of stimulus and response pairings. In our manipulation, we strengthen one of these incongruent pairings through the formation of an implementation intention using an "if...then" format. It may be that this representation or "event file" experiences a higher activation (or perhaps lower threshold) through the use of this self-regulatory technique. When one member of this association is triggered, it tends to activate the other member. The fact that an intentional self-regulatory technique was successful in reducing the Simon effect for the S-R pairing specified in the implementation intention has implications for theory building. Our result is more in line with the explanation of the Simon effect offered by Hommel et al. (2004) regarding event files rather than an explanation relying on suppression or gating mechanisms. Our results imply that participants were not suppressing the influence of spatial location but rather that the incongruent pairing of a stimulus + response was strengthened by encoding an implementation intention

Other studies have demonstrated that the Simon effect can be reduced (e.g., Tagliabue et al., 2000) or even reversed (e.g., Proctor \& Lu, 1999) through the use of task instructions and/or practice. According to a computational model proposed by Tagliabue et al. (2000), long-term links between the stimulus and response positions pre-exist before the experiment. Simon task instructions can be constructed to create short-term links between stimulus and responses positions leading to a modification of performance such that the long-term links are overridden by the shortterm associations. If we consider the current findings in the context of this model, implementation intentions provide an alternative way to modify the short-term S$\mathrm{R}$ links. In contrast to previous studies (Proctor \& Lu, 
1999; Tagliabue et al., 2000), implementation intentions reduced the Simon effect through one act of encoding as opposed to the need to use multiple practice trials. Furthermore, the manipulation was very specific in the sense that it only influenced the stimulus that was specified within the implementation intention. An interesting question for further research is to investigate how long-lasting are these influences. Furthermore, it would be important to examine whether implementation intentions can not only reduce the Simon effect but lead to a reversal of it.

But why are implementation intentions effective? First, encoding an implementation intention leads to a heightened accessibility of the situational cue helping to facilitate the detection of that cue in the environment. In a sense, they create some kind of perceptual readiness. Second, implementation intentions establish a situation-behavior link and in turn established routines linked to a relevant context release the critical goal-directed behaviour once the situational cue is encountered. However, there may be a downside to forming implementation intentions such that competing opportunities to act may not receive the same amount of attention. For example, in the task-switching paradigm the heightened activation of the critical stimulus ("E") led to facilitation but this effect was not found for the remaining neutral stimuli in this condition. As stated earlier, implementation intentions is a very specific manipulation which benefits only the critical cue that was specified in the formation of the implementation intention. In the Simon task in Experiment 2, a similar trade-off effect was found. Participants made more errors for congruent $S-R$ pairings in the implementation intention trials compared to the goal intention trials. Therefore, the increased activation of the incongruent $\mathrm{S}-\mathrm{R}$ pairing may have led subjects to focus on the incongruent stimuli and thereby led to some neglecting of the congruent stimuli. Thus, there may be some attention-related costs caused by forming implementation intentions in this task.

\section{Conclusion}

The focus of the current research was to examine the influence of a self-regulatory strategy in two different task domains that are known to require high levels of executive control. In Experiment 1, forming implementation intentions in the context of a task-switching paradigm led to an overall reduction of switch costs in the implementation intention condition relative to the goal condition. In Experiment 2, forming implementation intentions reduced the effects of spatial location in a
Simon task specifically for the stimulus that was stated within the implementation intention. We therefore conclude that performing tasks that require high levels of executive control can be facilitated if task performance is prepared in advance by making if-then plans that specify how one responds to that stimulus.

\section{References}

Aarts, H., Dijksterhuis, A., \& Midden, C. (1999). To plan or not to plan? Goal achievement of interrupting the performance of mundane behaviors. European Journal of Social Psychology, 29, 971-979.

Allport, D. A., Styles, E. A., \& Hsieh, S. (1994). Shifting intentional set: Exploring the dynamic control of tasks. In C. Umilta \& M. Moscovitch (Eds.), Attention and performance $X V$ : Conscious and nonconscious information processing (pp. 421-452). Hillsdale, NJ: Erlbaum.

Bandura, A. (1986). Social foundations of thought and action: A social cognitive theory. Englewood Cliffs, NJ: Prentice Hall.

Bargh, J. A. (1989). Conditional automaticity: Varieties of automatic influence in social perception and cognition. In J. S. U1eman, \& J. A. Bargh (Eds.), Unintended thought (pp. 3-51). London: Guilford Press.

Bargh, J. A. (1994). The four horsemen of automaticity: Awareness, intention, efficiency, and control in social cognition. In R. S. Wyer, Jr. \& T. K. Srull (Eds.), Handbook of social cognition, Vol. 1: Basic processes (2nd ed.) (pp. 1-40). Hillsdale, NJ: Erlbaum.

Bargh, J. A., Gollwitzer, P. M., Lee-Chai, A. L., Barndollar, K., \& Troetschel, R. (2001). The automated will: Nonconscious activation and pursuit of behavioral goals. Journal of Personality and Social Psychology, 81, 1014-1027.

Broadbent, D. E. (1958). Perception and communication. London: Pergamon Press.

Chasteen, A. L., Park, D. C., \& Schwarz, N. (2001). Implementation intentions and facilitation of prospective memory. Psychological Science, 12, 457-461.

Cohen, A.-L., Jaudas, A., \& Gollwitzer, P. M. (2005). Working memory load and self-regulatory strategies modulate the cost of remembering to remember. Paper presented at the 2 nd meeting of the international conference on prospective memory, Zurich, Switzerland.

De Jong, R., Liang, C.-C., \& Lauber, E. (1994). Conditional and unconditional automaticity: A dual-process model of effects of spatial stimulus-response correspondence. Journal of Experimental Psychology: Human Perception \& Performance, 20, 731-750.

Dreisbach, G., Haider, H., \& Kluwe, R. H. (2002). Preparatory processes in the task-switching paradigm: Evidence from the use of probability cues. Journal of Experimental Psychology: Learning, Memory, and Cognition, 28, 468-483.

Gollwitzer, P. M. (1993). Goal achievement: The role of intentions. European Review of Social Psychology, 4, 141-185.

Gollwitzer, P. M. (1999). Implementation intentions: Strong effects of simple plans. American Psychologist, 54, 493-503.

Gollwitzer, P. M., \& Schaal, B. (1998). Metacognition in action: The importance of implementation intentions. Personality and Social Psychology Review, 2, 124-136.

Gollwitzer, P. M., \& Sheeran, P. (2006) . Implementation intentions and goal achievement: A meta-analysis of effects and processes. Advances in Experimental Social Psychology, 38, 69-119. 
Hahn, S., Andersen, G. J., \& Kramer, A. F. (2003). Multidimensional set switching. Psychonomic Bulletin \& Review, 10, 503-509.

Hommel, B. (1993). Inverting the Simon effect intention: Determinants of direction and extent of effects of irrelevant spatial information. Psychological Research/Psychologische Forschung, 55, 270-279.

Hommel, B. (1997). Interactions between stimulus-stimulus congruence and stimulus-response compatibility. Psychological Research Psychologische Forschung, 59, 248-260.

Hommel, B., Proctor, R. W., \& Vu, K-P. L. (2004). A featureintegration account of sequential effects in the Simon task. Psychological Research/Psychologische Forschung, 68, 1-17.

Kornblum, S., Hasbroucq, T., \& Osman, A. (1990). Dimensional overlap: Cognitive basis for stimulus-response compatibilityA model and taxonomy. Psychological Review, 97, 253-270.

Lu, C-H., \& Proctor, R. W. (1995). The influence of irrelevant location information on performance: A review of the Simon and spatial Stroop effects. Psychonomic Bulletin \& Review, 2 , 174-207.

Mayr, U., \& Kliegl, R. (2003). Differential effects of cue changes and task changes on task-set selection costs. Journal of Experimental Psychology: Learning, Memory, and Cognition, 29, 362-372.

Meiran, N., \& Chorev, Z. (2005). Phasic alertness and the residual task-switching cost. Experimental Psychology, 52, 109-124.

Monsell, S. (2003). Task switching. Trends in Cognitive Sciences, 7, 134-140.

Mordkoff, J. T. (1998). Between-dimension flanker effects: A clarification with encouraging implications. Psychonomic Bulletin \& Review, 5, 670-675.

Proctor, R. W., \& Lu, C-H. (1999). Processing irrelevant location information: Practice and transfer effects in choice-reaction tasks. Memory \& Cognition, 27, 63-77.

Ratcliff, R. (1979). Group reaction time distributions and an analysis of distribution statistics. Psychological Bulletin, 86, 446-461.

Rogers, R. D., \& Monsell, S. (1995). Costs of a predictable switch between simple cognitive tasks. Journal of Experimental Psychology: General, 124, 207-231.

Schneider, W. (1988). Micro-Experimental Laboratory: An integrated system for IBM PC compatibles. Behavior Research Methods, Instruments, \& Computers, 20, 206-217.
Shallice, T. (2005). The fractionation of supervisory control. In M. S. Gazzaniga (Ed.).The cognitive neurosciences III. Cambridge, MA: MIT Press.

Simon, J. R. (1990). The effects of an irrelevant directional cue on human information processing. In R. W. Proctor \& T. G. Reeve (Eds.), Stimulus-resopnse compatibility: An integrated perspective (pp. 31-86). Amsterdam: North-Holland.

Sohn, M-H., \& Anderson, J. R. (2001). Task preparation and task repetition: Two-component model of task switching. Journal of Experimental Psychology: General, 130, 764-778.

Stürmer, B., Leuthold, H., \& Sommer, W. (1998). Strategische Effeckte und kurzfristige Modulationen im Simon-Paradigma [Strategic effects and short-term modulations in the Simon paradigm] [Abstract]. In H. Lachnit, A. Jacobs, \& F. Rösler (Eds.), Experimentelle Psychologie: Abstracts der 40. Tagung experimentellareitender Psychologen (pp. 365-366). Lengerich: Pabst Science Publishers.

Stürmer, B., Leuthold, H., Soetens, E., Schröter, H., \& Sommer, W. (2002). Control over location-based response activation in the Simon task: Behavioral and electrophysiological evidence. Journal of Experimental Psychology: Human Perception and Performance, 28, 1345-1363.

Tagliabue, M., Zorzi, M., Umiltà, C., \& Bassignani, F. (2000). The Role of Long-Term-Memory and Short-Term-Memory Links in the Simon Effect. Journal of Experimental Psychology: Human Perception and Performance, 26, 648-670.

Vu, K-P. L., Proctor, R. W., \& Urcuioli, P. (2003). Transfer effects of incompatible location-relevant mappings on a subsequent visual or auditory Simon task. Memory \& Cognition, 31, $1146-1152$.

Waszak, F., Hommel, B., \& Allport, A. (2003). Task-switching and long-term priming: Role of episodic stimulus-task bindings in task-shift costs. Cognitive Psychology, 46, 361-413.

Webb, T. L., \& Sheeran, P. (2003). Can implementation intentions help to overcome ego-depletion? Journal of Experimental Social Psychology, 39, 279-286.

Wylie, G., \& Allport, A. (2000). Task switching and the measurement of "switch costs." Psychological research/Psychologische Forschung. Special Executive Control, 63, 212-233. 